\title{
Furkert, Matthias (2015): Erkennen und Handeln: Restrukturierung der landesplanerischen Mittelbereiche in Rheinland-Pfalz
}

\author{
Hamburg: Kovač. XXVII, 485 Seiten. Geographica, Schriftenreihe Geowissenschaften \\ und Geographie, Band 11
}

\section{Hans H. Blotevogel ${ }^{1}$}

Online publiziert: 1. November 2016

(c) Springer-Verlag Berlin Heidelberg 2016

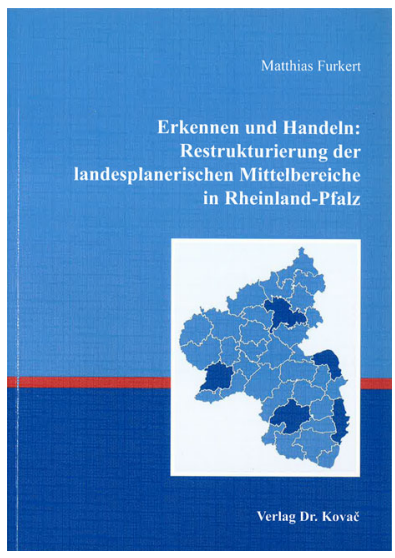

Die Aussage, dass das Zentrale-Orte-Konzept nach wie vor zu den tragenden Säulen der Raumordnung in Deutschland und Österreich gehört, dürfte zumindest in der deutschsprachigen Raumordnungs-Community auf eine breite Zustimmung stoßen. Dieses Statement findet sich auch in dem vorliegenden voluminösen Buch, das auf eine Dissertation im Fach Geographie an der Universität Trier von 2014 zurückgeht. Tatsächlich weisen - abgesehen von den Stadtstaaten - sämtliche Bundesländer Deutschlands und Österreichs in ihren Raumordnungsplänen Zentrale Orte unterschiedlicher Stufen aus. Allerdings unterscheiden sich die Zielsetzungen, Definitionen und Darstellungsformen dieses Steuerungsinstruments in der Raumordnungspraxis der Bundesländer erheblich und mit den sukzessiv erfolgenden Novellierungen der Raumordnungspläne nehmen die Diver-

Prof. Dr. Hans H. Blotevogel

hans.blotevogel@univie.ac.at

1 Institut für Geographie und Regionalforschung, Universität Wien, Universitätsstraße 7, 1010 Wien, Österreich

genzen immer mehr zu. Teils werden die Zentralen Orte auf die Versorgung mit Funktionen der öffentlichen Daseinsvorsorge bezogen, teils werden der Einzelhandel und/oder die Arbeitsmarktfunktion einbezogen. Teils werden ganze Gemeinden in ihrer territorialen Erstreckung als Zentrale Orte ausgewiesen, teils nur die zentralen Siedlungsbereiche. Teils beschränken sich die Darstellungen in den Plänen auf die Klassifikation der Gemeinden bzw. Zentralen Orte nach zwei oder drei Hierarchiestufen, teils werden auch Verflechtungsbereiche ausgewiesen. Die zunehmenden Divergenzen führen dazu, dass beispielsweise ein Mittelzentrum im Bundesland A nicht mehr dasselbe meint wie im Bundesland B, sodass nicht nur die Vergleichbarkeit, sondern auch die Rechtssicherheit in Verwaltungsgerichtsverfahren massiv beeinträchtigt wird. Insofern ist dringend geboten, das Instrument des Zentrale-Orte-Konzepts möglichst bundesweit einheitlich zu normieren und seine Implementation in den Raumordnungsplänen auf eine wissenschaftlich fundierte und schlüssig begründete Basis zu stellen.

Hier setzt die Arbeit von Matthias Furkert an, indem er einen konkreten Vorschlag für die Überarbeitung der mittelzentralen Ebene in Rheinland-Pfalz erarbeitet. Die Fokussierung auf diese Ebene ist insofern gerechtfertigt, als den Mittelzentren eine herausragende Rolle für die über die kommunale Grundversorgung hinausgehende Daseinsvorsorge, vor allem in den Bereichen Bildung, Gesundheitswesen und Verkehr, zukommt. Unmittelbarer Anlass sind die anstehende Fortschreibung des rheinland-pfälzischen Landesentwicklungsplans und die Notwendigkeit, die in Anbetracht des demographischen Wandels und fiskalischer Engpässe völlig unrealistisch große Zahl von Mittelzentren zu reduzieren.

Matthias Furkert entwirft einen weiten Argumentationsbogen, indem er zunächst eine wissenschaftstheoretische Grundlegung vornimmt. Er favorisiert die Wissenschafts- 
philosophie des Pragmatismus nach J. Dewey und C. S. Peirce (nicht Pierce, wie Furkert irrtümlicherweise schreibt) als methodologische Basis. Diese Entscheidung ist für eine politikberatende Studie durchaus sinnvoll, weil es im Pragmatismus nicht wie in den Naturwissenschaften um epistemologische Wahrheit, sondern um praktische Zweckmäßigkeit geht. Allerdings irritiert ein wenig nicht nur, dass Furkert wiederholt von einer ,Pragmatismustheorie“ schreibt, sondern auch die „Abduktion“ als logisches Argumentationsmodell hervorhebt, denn in der neueren Wissenschaftsphilosophie wird dieses eher kritisch (und allenfalls als Prinzip einer Ökonomie der Hypothesenbildung) gesehen. Überhaupt hätte die gesamte Arbeit auch ohne diese verhältnismäßig weit ausholende wissenschaftstheoretische Verortung auskommen und sich auf einen knappen Verweis beschränken können.

Kapitel 3 skizziert die Rahmenbedingungen, vor denen die Überarbeitung der landesplanerischen Mittelbereiche steht. Furkert betont vor allem die demographischen Entwicklungen, welche in ländlichen Regionen die Mindesttragfähigkeiten unterschreiten, die prekäre fiskalische Entwicklung und die aktuelle Diskussion um das Postulat einer flächendeckenden Gleichwertigkeit. Konkreter wird dann Kapitel 4, in dem verschiedene zentralörtliche Restrukturierungsvarianten wie Funktionsteilungen und mehrkernige Funktionsräume diskutiert werden. Kapitel 5 beschreibt das Forschungsdesign. Die Argumentation basiert neben der Auswertung von Dokumenten vor allem auf 34 Experteninterviews mit Vertreterinnen und Vertretern der Raumordnungsverwaltungen, der Finanzverwaltung und des Städtetags sowie auf Auswertungen einschlägiger Statistiken zur Zentrenausstattung und zu Pendlerverflechtungen. In Kapitel 6 wird anhand von Berlin-Brandenburg, Niedersachsen und Sachsen ein Überblick über aktuelle Fortentwicklungen der zentralörtlichen Systeme in anderen Bundesländern gegeben. Eindrücklich wird aufgezeigt, wie unterschiedlich die Länder mit dem Instrument Zentrale-Orte-Konzept umgehen.

Interessante Befunde enthält auch Kapitel 7, in dem Einschätzungen zur Restrukturierung des rheinland-pfälzischen Zentrale-Orte-Konzepts aus der Sicht des Finanzministeriums, des Städtetages sowie der Landes- und Regionalplanung referiert werden. Deutlich wird die prinzipielle Akzeptanz des Konzepts sowohl für den kommunalen Finanzausgleich als auch für die Infrastrukturplanung - allerdings liegt das wohl eher an der Auswahl der Befragten, denn die betroffenen Fachplanungen wurden nicht in das Sample der Interviewpartner einbezogen! Auch über die Notwendigkeit einer realistischen Reduktion der Mittelzentren sind sich die befragten Experten prinzipiell einig, während bei den Fragen von funktionsteiligen Zentren und polyzentralen Funktionsräumen die Meinungen deutlich auseinandergehen.
Eine datengestützte Analyse der (potenziellen) Mittelzentren und ihrer Verflechtungsbereiche hinsichtlich der aktuellen und künftigen Tragfähigkeiten erfolgt in Kapitel 8. Vor allem für die Funktionsbereiche Bildung, Gesundheitswesen und Verkehr/Erreichbarkeit werden die derzeit landesplanerisch ausgewiesenen Mittelzentren einer detaillierten Ausstattungs- und Tragfähigkeitsanalyse unterzogen. Wenig überraschend zeigt sich, dass vor allem in den dünner besiedelten ländlichen Räumen der Eifel, des Westerwaldes, des Hunsrücks und der Westpfalz viele landesplanerische Mittelzentren und ihre Bereiche nicht über eine angemessene Ausstattung bzw. über eine hinreichende Bevölkerungstragfähigkeit verfügen. Problematisch sind einzelne Bezeichnungen der ansonsten gut nachvollziehbaren Analyse. So ist ständig nicht nur von „Unterversorgung“, sondern auch von „Überversorgung“ die Rede. Damit entsteht der Eindruck, es sei ein legitimes Ziel der Landesplanung, die Versorgungsniveaus auf einen statistischen Mittelwert zu nivellieren. Tatsächlich kann es doch nur darum gehen, politisch nicht akzeptable Versorgungsdefizite auszugleichen, wobei die Akzeptanz von Versorgungsdefiziten politisch definiert werden müsste und nicht einfach aus Verteilungsstatistiken abgeleitet werden kann. Eine Ungenauigkeit besteht auch darin, dass Furkert Beschäftigte in Prozent der Wohnbevölkerung darstellt, weil es sich dabei nicht um Anteilswerte, sondern um Verhältniswerte bzw. Besatzziffern handelt.

Kapitel 9 enthält einen konkreten Restrukturierungsvorschlag. Aufgrund der Kriterien Ausstattung, Tragfähigkeit und Erreichbarkeit empfiehlt Furkert eine Reduktion der Mittelzentren und Mittelbereiche um ungefähr ein Drittel. Durch diesen sachlich gut begründeten Vorschlag würde sich die rheinland-pfälzische Landesplanung von der Illusion verabschieden, trotz mangelnder Tragfähigkeiten ein unfinanzierbares, dichtes Netz von Mittelzentren ausbauen zu können. Solche „Placebo-Zentralorte“, die häufig auf Druck lokaler politischer Interessen in die Raumordnungspläne aufgenommen werden, aber niemals in die Realität umgesetzt werden können, beeinträchtigen die Steuerungsfähigkeit des Zentrale-Orte-Konzepts, weil damit die Auswahlkriterien überdehnt werden, die Vergleichbarkeit der Funktionszuweisung nicht mehr gegeben ist und die Justiziabilität der Planung prekär wird. Da in dünn besiedelten Gebieten der Eifel, des Westerwaldes und des Hunsrücks durch die konsequente Anwendung der Schwellenwerte teilweise Erreichbarkeitsdefizite entstehen, schlägt Furkert in den Fällen Adenau, Altenkirchen, Dierdorf, Hermeskeil, Meisenheim, Neuerburg und St. Goar/St. Goarshausen sogenannte mittelzentrale Raumstrukturzentren vor, welche trotz mangelnder Tragfähigkeit als mittelzentrale Ankerzentren einer besonderen Stützung bedürfen. Im Umland der fünf rheinland-pfälzischen Oberzentren Mainz, Ludwigshafen, Kaiserslautern, Koblenz und Trier schlägt Furkert 
vor, auf die Ausweisung gesonderter Mittelbereiche zu verzichten und stattdessen „Funktionsräume“ auszuweisen, in denen jeweils mehrere Mittelzentren existieren, jedoch wegen der vielfachen Überlappungen der Verflechtungen keine gesonderten Mittelbereiche abgrenzbar sind. Damit wird die Implementation des Zentrale-Orte-Konzepts zweigeteilt: In den eher ländlichen Räumen werden entsprechend dem traditionellen Modell mono- bzw. (in Ausnahmefällen auch) funktionsteilige Mittelzentren mit linear abgegrenzten Bereichen vorgeschlagen. Hingegen favorisiert Furkert für die Stadtregionen der fünf Oberzentren zwar ebenfalls Mittelzentren, doch liegen diese zusammen mit dem jeweiligen Oberzentrum in polyzentralen „Funktionsräumen“ ohne weitere innere Bereichsgliederung.

Inwieweit diese Vorschläge in die Praxis umgesetzt werden können, erörtert Furkert im Kapitel 10. Anhand einer SWOT-Analyse werden Argumente pro und contra diskutiert und drei unterschiedliche Implementationsstrategien für die Weiterentwicklung des mittelzentralen Systems vorgeschlagen.

Das umfangreiche Werk ist nicht gerade eine leichte Lektüre, doch erleichtern Zwischen-Resümees die Orientie- rung. Die Arbeit zeigt aber auch, mit welcher Komplexität die Weiterentwicklung des Zentrale-Orte-Konzepts verbunden ist, wenn die Argumentation wissenschaftlich fundiert und zugleich praxisorientiert im Sinne einer Umsetzbarkeit in die politisch-planerische Realität der Raumordnung sein soll. Man muss nicht alle Vorschläge überzeugend finden. So dürften vor allem die polyzentralen Funktionsräume, in denen keine Mittelbereiche unterschieden werden, kontrovers diskutiert werden. Auch aus wissenschaftlicher Sicht ist hier eher Skepsis anzumelden, zumal solche polyzentralen Gebilde im kommunalen Finanzausgleich kaum handhabbar sein dürften. Unstrittig und unbedingt zu begrüßen ist jedoch der Vorschlag einer drastischen Reduktion der bisher inflationär ausgewiesenen Mittelzentren. Wenn das Zentrale-Orte-Konzept tatsächlich eine Steuerungswirkung entfalten soll, muss die Landesplanung auf die Ausweisung unrealistischer Phantom-Zentralorte verzichten. Wie auch Furkert hervorhebt, gibt es gute Gründe, das Steuerungsinstrument Zentrale-Orte-Konzept so zu schärfen, dass es nicht - wie bisher häufig - nur noch wirkungslose Symbolpolitik ist. 